\title{
ARTICLE OPEN Prediction of pressure-promoted thermal rejuvenation in metallic glasses
}

\author{
Narumasa Miyazaki ${ }^{1}$, Masato Wakeda ${ }^{1}$, Yun-Jiang Wang ${ }^{2}$ and Shigenobu Ogata ${ }^{1,3}$
}

Rejuvenation is the structural excitation of glassy materials, and is a promising approach for improving the macroscopic deformability of metallic glasses. This atomistic study proposes the application of compressive hydrostatic pressure during the glass-forming quenching process and demonstrates highly rejuvenated glass states that have not been attainable without the application of pressure. Surprisingly, the pressure-promoted rejuvenation process increases the characteristic short- and mediumrange order, even though it leads to a higher-energy glassy state. This 'local order'-'energy' relation is completely opposite to conventional thinking regarding the relation, suggesting the presence of a well-ordered high-pressure glass/high-energy glass phase. We also demonstrate that the rejuvenated glass made by the pressure-promoted rejuvenation exhibits greater plastic performance than as-quenched glass, and greater strength and stiffness than glass made without the application of pressure. It is thus possible to tune the mechanical properties of glass using the pressure-promoted rejuvenation technique.

npj Computational Materials (2016) 2, 16013; doi:10.1038/npjcompumats.2016.13; published online 24 June 2016

\section{INTRODUCTION}

Rejuvenation is the opposite phenomena to ageing, and it has been investigated for decades in relation to molecular and polymeric glasses, ${ }^{1}$ and more recently in relation to metallic glasses (MGs). ${ }^{2-8}$ In contrast to rejuvenation, ageing is a structural relaxation of the glassy material that changes both the internal structure and mechanical properties of the glass. Well-aged glass usually exhibits brittle fracture triggered by shear band formation ${ }^{9}$ at an ambient temperature and usual strain rate. As brittle fracture is a fatal flaw in terms of the application of MGs as structural and mechanical materials, realising a highly rejuvenated state without losing strength and stiffness, and preventing both ageing and brittle fracture (i.e., maintaining the highly rejuvenated state for a long time) are key factors in expanding the applicability of MGs. Some mechanical approaches, such as the application of highpressure torsion $^{3}$ and shot peening ${ }^{5}$ to mechanically realise a rejuvenated state in MGs, have been proposed, but significant shape change or heterogeneous property distribution is unavoidable. Recently, thermal-based approaches such as thermal rejuvenation consisting of recovery annealing ${ }^{10}$ conducted above the glass transition temperature $T_{\mathrm{g}}$ followed by fast cooling have been proposed. ${ }^{2,6}$ The thermal approach can control not only the surface properties but also the interior of any glass component shape. It can be also utilised to maintain plastic performance in thermoplastic nanoimprinting of $\mathrm{MG}^{11}$ which has been developed to realise low-cost fabrication of micro- and nano-sized devices. However, thermal rejuvenation requires rapid cooling after annealing, which should be faster than that of the initial glassforming quenching process, and moreover it may lose intrinsic strength and elastic stiffness. ${ }^{2,6}$ These are serious drawbacks in terms of practical application of this approach. To overcome the practical drawbacks, we propose a pressure-promoted thermal rejuvenation technique here, in which pressure is applied during the glass-forming quenching process.

In this study, we performed molecular dynamics (MD) simulations that demonstrate the feasibility of the proposed pressure-promoted thermal rejuvenation process, and we discuss the underlying physics based on diffusivity, a-relaxation, $T_{\mathrm{g}}$ and internal structure analyses. We found that pressure-promoted rejuvenation is achieved only when a comprehensive influence of three pressure effects on glass-forming process, i.e., effects of temperature-dependent liquid inherent structure energy, pressure-dependent diffusively slowdown (relaxation slowdown) and $T_{\mathrm{g}}$ increase, gains ascendancy over an increase in the Arrhenius-non-Arrhenius diffusivity transition temperature. Then, using MD uniaxial tensile test, we demonstrate that the pressurepromoted thermally rejuvenated glass actually has a weak strain localisation tendency, resulting in better plastic performance. We also examine the feasibility of the pressure-promoted thermal rejuvenation on various alloy systems. Note that some of the examined alloy systems do not exhibit pressure-promoted thermal rejuvenation because the comprehensive influence of three pressure effects fails to override the diffusivity transition temperature rise effect in these alloy systems.

\section{RESULTS \\ Prediction of pressure-promoted thermal rejuvenation map \\ We constructed MG models via a melt-quenching process $(A \rightarrow D$ in Figure 1a) as follows. First, the molten alloy was equilibrated at $3,000 \mathrm{~K}$ for $100 \mathrm{ps}(\mathrm{A} \rightarrow \mathrm{B})$ with zero external pressure; then, the model was quenched to $0 \mathrm{~K}$ at the same constant cooling rate of $K=1.0 \mathrm{~K} / \mathrm{ps}(\mathrm{B} \rightarrow \mathrm{C})$. Next, the potential energy was minimised using the steepest decent (SD) method at $0 \mathrm{~K}$ under zero pressure $P=0 \mathrm{GPa}(\mathrm{C} \rightarrow \mathrm{D})$. The constructed MG model (state $\mathrm{D})$ is here}

\footnotetext{
${ }^{1}$ Department of Mechanical Science and Bioengineering, Graduate School of Engineering Science, Osaka University, Osaka, Japan; ${ }^{2}$ State Key Laboratory of Nonlinear Mechanics,

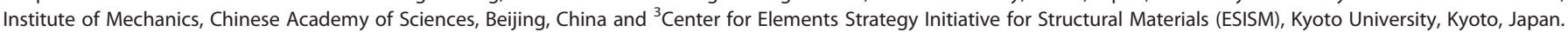
Correspondence: M Wakeda (wakeda@me.es.osaka-u.ac.jp) or S Ogata (ogata@me.es.osaka-u.ac.jp)

Received 4 November 2015; revised 25 April 2016; accepted 17 May 2016
} 


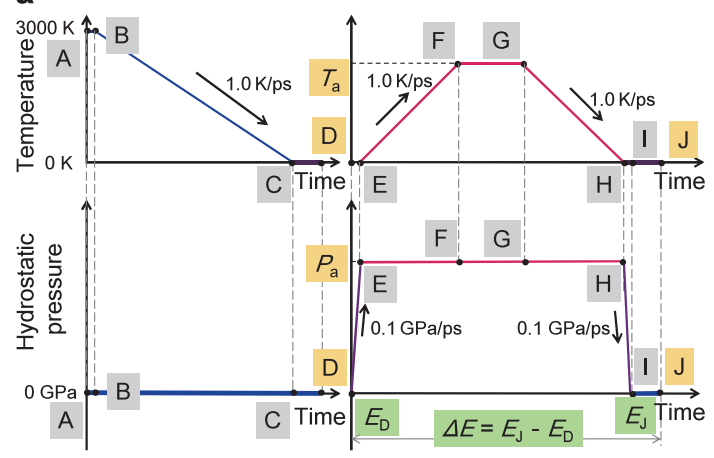

b

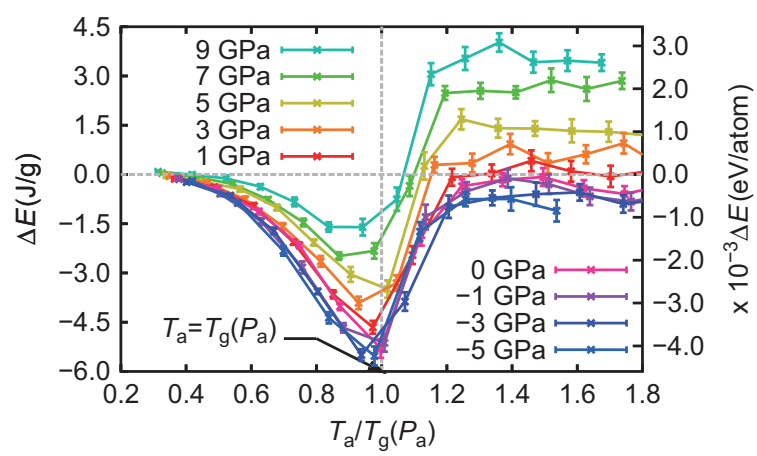

Figure 1. (a) Schematic illustration of initial melt-quenching $(A \rightarrow D)$ and subsequent thermal-pressure loading $(D \rightarrow J)$ processes. (b) The change in potential energy $\Delta E$ of the $\mathrm{Zr}_{50} \mathrm{Cu}_{40} \mathrm{Al}_{10}$ model induced by the thermal-pressure loading process $(\mathrm{D} \rightarrow \mathrm{J})$. In $\mathbf{b}$, the error bars represent the s.e. of 10 different simulations. The positive and negative pressures indicate compressive and tensile pressures, respectively. The horizontal axis represents the annealing temperature, which is normalised by $T_{\mathrm{g}}\left(P_{\mathrm{a}}\right)$ for each pressure condition. In general, $T_{\mathrm{g}}$ depends on the applied pressure condition. ${ }^{31}$ Therefore, we conducted melt-quenching simulations under different pressure conditions, and computed $T_{\mathrm{g}}\left(P_{\mathrm{a}}\right)$ for each $P_{\mathrm{a}}$ from the kink in the quenching process volume-temperature curve (Supplementary Figures S1 and S2).

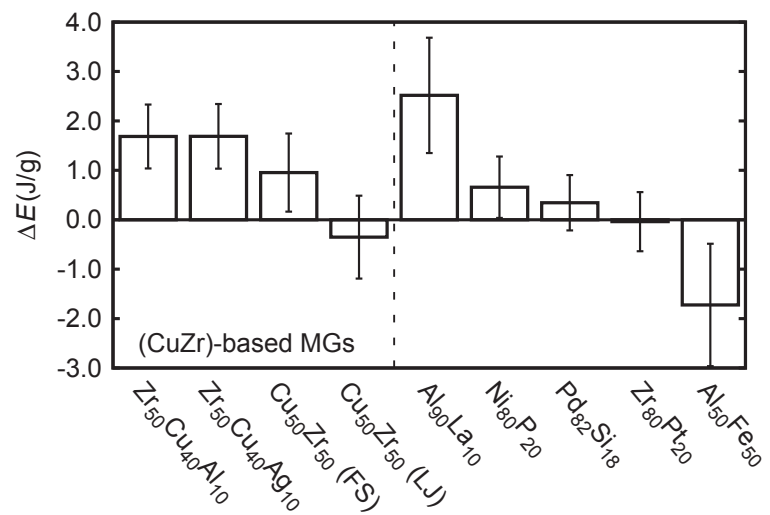

Figure 2. The change in potential energy $\Delta E$ of $\mathrm{Zr}_{50} \mathrm{Cu}_{40} \mathrm{Al}_{10}$, $\mathrm{Zr}_{50} \mathrm{Cu}_{40} \mathrm{Ag}_{10}, \mathrm{Cu}_{50} \mathrm{Zr}_{50}(\mathrm{FS}), \mathrm{Cu}_{50} \mathrm{Zr}_{50}(\mathrm{LJ}), \mathrm{Al}_{90} \mathrm{La}_{10}, \mathrm{Ni}_{80} \mathrm{P}_{20}, \mathrm{Pd}_{82} \mathrm{Si}_{18}$ $\mathrm{Zr}_{80} \mathrm{Pt}_{20}$ and $\mathrm{Al}_{50} \mathrm{Fe}_{50}$. For each alloy system, we conducted 10 independent simulations with different initial atomic configurations and velocities. The averaged $\Delta E$ values over 10 simulations with s.e. bars are shown. Similar to Figure $1 \mathrm{~b}$, detailed analyses of effect of annealing temperature and pressure were conducted for $\mathrm{Cu}_{50} \mathrm{Zr}_{50}$ (LJ) model (Supplementary Figure S5).

referred to as the 'as-quenched model.' Using the as-quenched model, we then conducted thermal-pressure loading simulations $(D \rightarrow J)$ comprising the following steps: (1) loading of the hydrostatic pressure up to the applied pressure $P_{\mathrm{a}}$ at $0 \mathrm{~K}(\mathrm{D} \rightarrow \mathrm{E})$; (2) heating from $0 \mathrm{~K}$ to the annealing temperature $T_{a}(E \rightarrow F)$; (3) isothermal annealing at $T_{\mathrm{a}}$ and $P_{\mathrm{a}}$ for $1 \mathrm{~ns}(\mathrm{~F} \rightarrow \mathrm{G})$; (4) cooling from $T_{\mathrm{a}}$ to $0 \mathrm{~K}(\mathrm{G} \rightarrow \mathrm{H})$ at a constant cooling rate of the $K=1.0 \mathrm{~K} / \mathrm{ps}$ as $B \rightarrow C$, followed by minimisation of the potential energy using the SD method; (5) unloading of all normal stresses $\left(\sigma_{x x}, \sigma_{y y}\right.$ and $\left.\sigma_{z z}\right)$ independently from $P_{\mathrm{a}}$ to $0 \mathrm{GPa}$ under zero velocity $(\mathrm{H} \rightarrow \mathrm{I})$; and (6) minimising the potential energy under zero pressure using the SD method $(I \rightarrow J)$. The final obtained model (state $J)$ will be hereafter referred to as the 'annealed model.' Cooling $(B \rightarrow C$ and $\mathrm{G} \rightarrow \mathrm{H}$ ) is conducted at a constant cooling rate of $K=1.0 \mathrm{~K} / \mathrm{ps}$ and heating rate $(E \rightarrow F)$ is also $1.0 \mathrm{~K} / \mathrm{ps}$. Pressure loading $(\mathrm{D} \rightarrow \mathrm{E})$ and unloading $(\mathrm{H} \rightarrow \mathrm{I})$ are conducted at a constant rate of $0.1 \mathrm{GPa} / \mathrm{ps}$. It is worth noting that the technique of thermal loading under high-pressure condition, such as $10 \mathrm{GPa}$ and more, has been already realised in experiments. ${ }^{12}$

In this study, we primarily use the $\mathrm{Zr}_{50} \mathrm{Cu}_{40} \mathrm{Al}_{10}$ MG model, where interatomic interactions are computed using the embedded atom method (EAM) potentials. ${ }^{13}$ Figure $1 \mathrm{~b}$ shows the change in the potential energy induced by the thermalpressure loading process $(\mathrm{D} \rightarrow \mathrm{J}) \Delta E=E_{J}-E_{\mathrm{D}}$, where $E_{\mathrm{D}}$ and $E_{\mathrm{J}}$ are the potential energies of the as-quenched and annealed models, respectively. $\Delta E$ represents the change in the glass state during the thermal-pressure loading process; $\Delta E$ becomes positive if rejuvenation occurs and falls below zero in the case of ageing.

Under zero external pressure $\left(P_{\mathrm{a}}=0 \mathrm{GPa}\right), \Delta E$ is solely induced by thermal loading. In Figure $1 \mathrm{~b}, \Delta E$ under the zero-pressure condition is negative below $1.3 T_{\mathrm{g}}$ and becomes almost zero above $1.3 T_{\mathrm{g}}$. This result demonstrates that rejuvenation cannot be realised under zero pressure if the quenching process $(G \rightarrow H)$ cooling rates are equal to those of the initial melt-quenching process $(B \rightarrow C)$. Our previous work ${ }^{2}$ demonstrated the feasibility conditions for rejuvenation induced by thermal loading, and the thermal rejuvenation was found to be realised only when $T_{\mathrm{a}}$ was above $1.1 T_{\mathrm{g}}$ and when the cooling rate after isothermal annealing was higher than that of the initial melt-quenching process. This was because the fast cooling suppresses ageing during the glass-forming quenching process. Thus, the $\Delta E$ profile under zero external pressure shown in Figure $1 \mathrm{~b}$ supports the conclusions of our previous work, ${ }^{2}$ even though the glass systems and employed interatomic potentials differ. On the other hand, $\Delta E$ profiles under 'non-zero' pressure conditions shift from those under zero pressure. The $\Delta E$ values for higher annealing temperatures $T_{\mathrm{a}}>1.1-1.2 T_{\mathrm{g}}$ clearly demonstrate that tensile pressure enhances ageing, whereas compressive pressure suppresses it. Thus, in this alloy system, the thermal rejuvenation is realised by the application of compressive external pressure instead of rapid quenching, but only when the annealing temperature is above the critical annealing temperature, $1.1-1.2 T_{\mathrm{g}}$. The degree of rejuvenation increases with increased compressive pressure and/or annealing temperature up to $\sim 1.3 T_{\mathrm{g}}$ implying that the employed compressive pressure has the same role in increasing the cooling rate in the heating-annealing-quenching process $(\mathrm{D} \rightarrow \mathrm{H})$ from a viewpoint of the degree of rejuvenation $\Delta E$. To clarify effect of the cooling rate $K$ of the processes $B \rightarrow C$ and $\mathrm{G} \rightarrow \mathrm{H}$ on $\Delta E$, we also conducted the same initial melt-quenching $(A \rightarrow D)$ and subsequent thermal-pressure loading simulations $(\mathrm{D} \rightarrow \mathrm{H})$ with the other several different cooling rates, such as $K=0.01, \quad 0.1$ and $10 \mathrm{~K} / \mathrm{ps}$ for $P_{\mathrm{a}}=5 \mathrm{GPa}, T_{\mathrm{a}}=1.3 T_{\mathrm{g}}\left(P_{\mathrm{a}}\right)$, and confirmed that thermal rejuvenation was actually realised in all the cooling rates tested here, and moreover no significant changes in $\Delta E$ can be found in the cases of cooling rate $K \leqslant 1.0 \mathrm{~K} / \mathrm{ps}$ (Supplementary Figure S3). It is worth noting that $\Delta E$ has the minimum value, in other words, ageing is the most striking, at around $T_{\mathrm{a}} \sim T_{\mathrm{g}}\left(P_{\mathrm{a}}\right)$ in Figure $1 \mathrm{~b}$. This is the result of a 

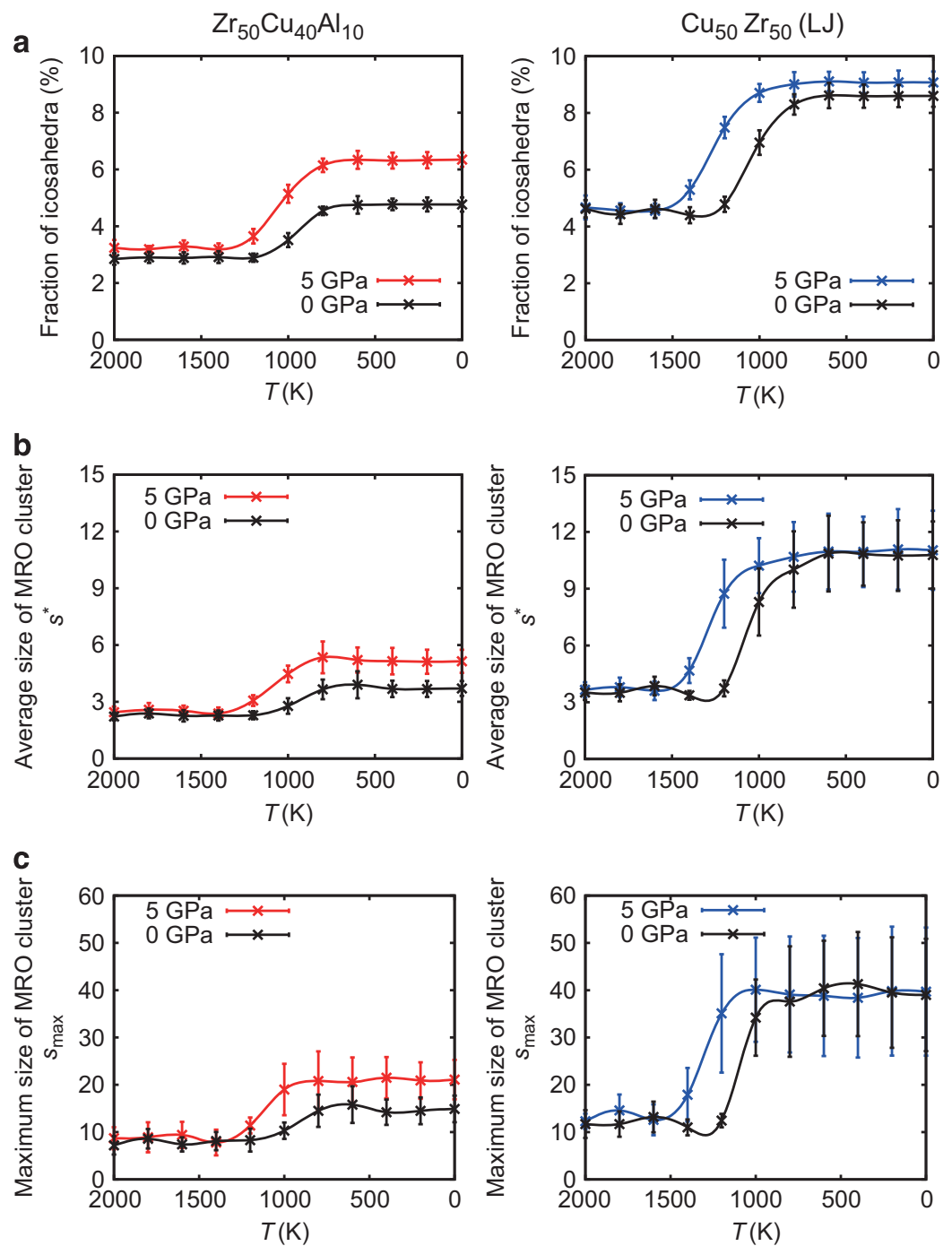

d
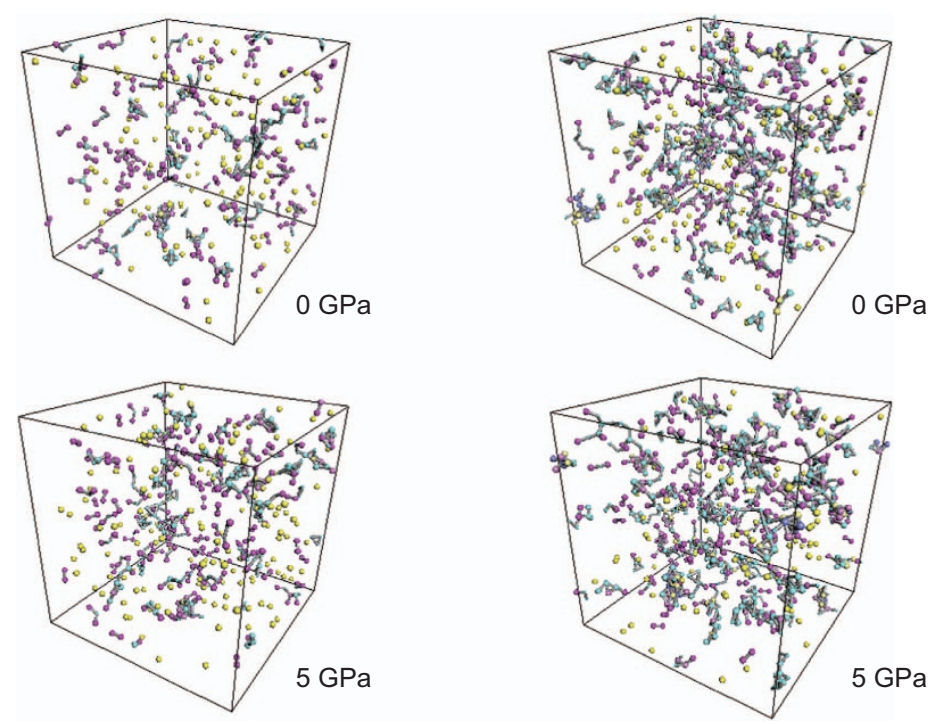

Figure 3. (a) Icosahedral $\mathrm{SRO}^{23}$ and (b, c) $\mathrm{MRO}^{24,25}$ exist in the inherent structure of the quenching process. The left figure shows the $\mathrm{Zr}_{50} \mathrm{Cu}_{40} \mathrm{Al}_{10}$ alloy results, whereas the right one shows the $\mathrm{Cu}_{50} \mathrm{Zr}_{50}(\mathrm{LJ})$ alloy results. (a) A fraction of icosahedral SRO, and (b, c) the average and maximum size of the MRO cluster composed of interpenetrating $s^{*}, s_{\max }$, respectively. The definitions of average and maximum size are given in ref. 25. (d) Spatial distribution of icosahedral SRO and $M R O^{24}$ in the $0 \mathrm{~K}$ inherent structure of the $\mathrm{Zr}_{50} \mathrm{Cu}_{40} \mathrm{Al}_{10}(\mathrm{left})$ and $\mathrm{Cu}_{50} \mathrm{Zr}_{50}$ (LJ; right) models constructed via the quenching process with $(P=5 \mathrm{GPa})$ and without pressure $(P=0 \mathrm{GPa})$. 
temperature dependency of two factors; (i) an allowable amount of relaxation (energy reduction) bounded by the energy state of equilibrium liquid, and (ii) an amount of relaxation during certain annealing time. The factor (i) decreases with increasing $T_{a}$, and rapidly decreases especially at above $T_{\mathrm{g}}$, whereas the factor (ii) increases with increasing $T_{a}$, and rapidly increases especially at above $T_{\mathrm{g}}$. As a result at $T_{\mathrm{a}} \sim T_{\mathrm{g}}\left(P_{\mathrm{a}}\right)$, the amount of ageing maximises because the relaxation rate is fast and moreover the large amount of relaxation is allowed (see a schematic illustration in Supplementary Figure S4).

To verify the feasibility of pressure-promoted thermal rejuvenation in other glass systems, we constructed eight different MG models via melt-quenching process $(A \rightarrow D)$ and applied the same thermal-pressure loading process $(D \rightarrow J)$ to the MG models, where the interatomic interactions were calculated from different interatomic potentials, such as $\mathrm{Zr}_{50} \mathrm{Cu}_{40} \mathrm{Ag}_{10}\left(\mathrm{EAM}^{14}\right), \mathrm{Cu}_{50} \mathrm{Zr}_{50}$ (Finnis-Sinclair: $\mathrm{FS}^{15}$ ), $\mathrm{Cu}_{50} \mathrm{Zr}_{50}$ (Lennard-Jones: $\mathrm{LJ}^{16}$ ), $\mathrm{Al}_{90} \mathrm{La}_{10}$ $\left(E M^{17}\right), \mathrm{Ni}_{80} \mathrm{P}_{20}\left(\mathrm{EAM}^{18}\right), \mathrm{Pd}_{82} \mathrm{Si}_{18}\left(\mathrm{EAM}^{19}\right), \mathrm{Zr}_{80} \mathrm{Pt}_{20}\left(\mathrm{EAM}^{20}\right)$ and $\mathrm{Al}_{50} \mathrm{Fe}_{50}\left(\mathrm{FS}^{21}\right)$. Here we set $P_{\mathrm{a}}=5 \mathrm{GPa}$ and $T_{\mathrm{a}}=1.3 T_{\mathrm{g}}\left(P_{\mathrm{a}}\right)$. The resultant changes in the potential energy $\Delta E$ are summarised in Figure 2. Pressure-promoted thermal rejuvenation can be seen in many of the examined alloy systems, whereas pressure-promoted 'ageing' can be observed in $\mathrm{Cu}_{50} \mathrm{Zr}_{50}(\mathrm{LJ}), \mathrm{Zr}_{80} \mathrm{Pt}_{20}$ and $\mathrm{Al}_{50} \mathrm{Fe}_{50}$. This fact reveals that the effect of pressure on the atomic diffusion, which dominates the structural relaxation in the quenching process, is strongly dependent on alloy composition; in other words, it depends on atomic sizes and interatomic interactions. Similar alloy dependence of pressure effects on structural relaxation has been reported for an experimental tensile test conducted on a notched MG sample. ${ }^{22}$

Changes in internal structure by compressive pressure

To reveal the internal structure change induced by compressive pressure, we analysed icosahedral short-range order $(\mathrm{SRO})^{23}$ and medium-range order (MRO) composed of interpenetrating icosahedra ${ }^{2,24,25}$ existing in the inherent structures of the quenching process from far above $T_{\mathrm{g}}$ to $0 \mathrm{~K}$, under a constant cooling rate $K$ and a constant pressure $P$. The inherent structure is defined as the structure of local minimum on the potential energy landscape at $P=0 \mathrm{GPa}$, and it is calculated using the following process: first, the potential energy from an instantaneous atomic configuration in the cooling process is minimised using the SD method. Then, the pressure is unloaded from $P$ to $0 \mathrm{GPa}$, and finally the potential energy is again minimised at $0 \mathrm{GPa}$. The results of the changes in internal structure are summarised in Figure 3, in which icosahedral SRO and MRO are surprisingly increased by compressive pressure in the case of $\mathrm{Zr}_{50} \mathrm{Cu}_{40} \mathrm{Al}_{10}$, and thus these ordered local structures are supersaturated and more tightly filled in by the compressive pressure application in cooling process, even though the compressive pressure leads to an energetically unstable (i.e., rejuvenated) glass state. These results reverse the conventional understanding of the relationship between characteristic topological order and energy state, in which much icosahedral SRO and MRO lead to an energetically more stable glass state and vice versa. ${ }^{23,24,26,27}$ Moreover, in the case of $\mathrm{Cu}_{50} \mathrm{Zr}_{50}(\mathrm{LJ})$, we cannot see a clear increase in the icosahedral SRO and MRO, even though the pressure leads to an energetically more stable (i.e., aged) glassy state (for other MG systems, see Supplementary Figure S6). These results suggest the presence of a well-ordered high-energy glassy state. It is worth noting that volume of the pressure-promoted thermally rejuvenated $\mathrm{Zr}_{50} \mathrm{Cu}_{40} \mathrm{Al}_{10}$ model with the compressive pressure application decreases with increasing the degree of rejuvenation $\Delta E$ (i.e., increasing the compressive pressure), and thus the volume is always smaller than that of as-quenched model (Supplementary Figure S7). On the other hand, the volume of thermally rejuvenated model with a rapid cooling rate of the $\mathrm{G} \rightarrow \mathrm{H}$ faster than that of the $B \rightarrow C$, without compressive pressure application (pure thermal rejuvenation model ${ }^{2}$ ), increases with increasing the $\Delta E$ (i.e., increasing the cooling rate of the $\mathrm{G} \rightarrow \mathrm{H}$ process), and thus the volume is always larger than the as-quenched model (Supplementary Figure S7). This means that although if these rejuvenated glasses have the same degree of rejuvenation $\Delta E$, these volumes are totally different; the former one has smaller and the latter one has larger volume than as-quenched model. More details of the volume and SRO analyses are plotted in Supplementary Figure S7. We show the schematic illustration of characteristics of glasses after the pure thermal rejuvenation and the pressure-promoted thermal rejuvenation in Figure 4, in which energy, density and SRO of as-quenched, pure thermally aged and rejuvenated, pressure-promoted thermally rejuvenated glasses are summarised.

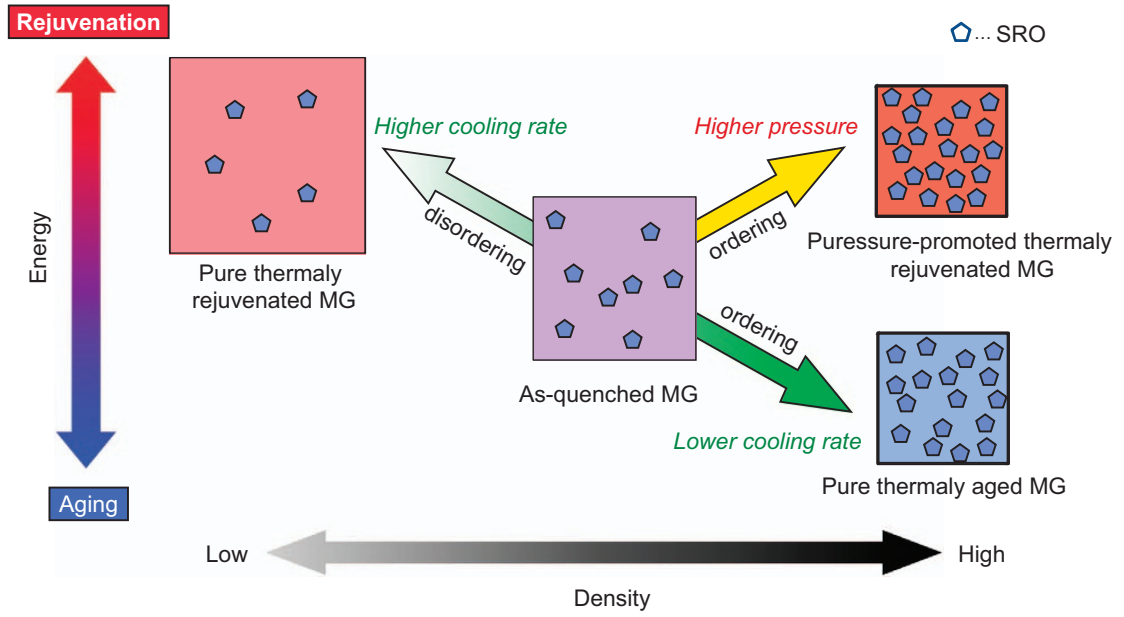

Figure 4. Schematic illustration of rejuvenation and ageing with and without pressure. The horizontal axis represents density, which increases form left to right, whereas the vertical axis represents energy, which increases from bottom up. The left, centre, right lower and right upper cartoons of glass structure represent pure thermally rejuvenated glass, as-quenched glass, pure thermally aged glass and pressure-promoted thermally rejuvenated glass, respectively. The pure thermally rejuvenated glass has lesser SRO and lower density, whereas the pure thermally aged glass has more SRO and higher density than the as-quenched glass. On the other hand, the pressure-promoted thermally rejuvenated glass has more SRO but higher density than the as-quenched glass. The colour of the glass cartoon represents energy state; blue shows lower energy, whereas red shows higher energy. 
a

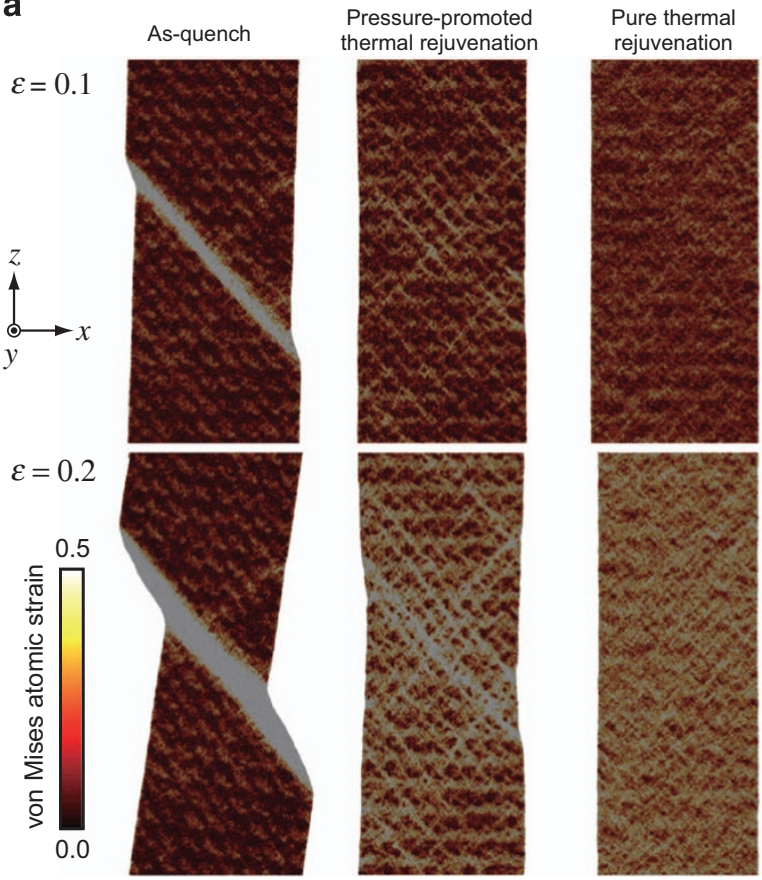

b

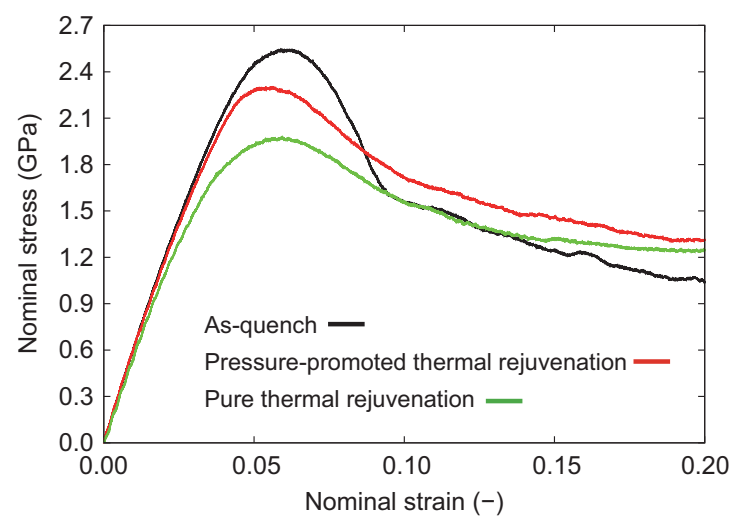

Figure 5. (a) von Mises atomic strain ${ }^{37}$ distributions in the uniaxal tensile tests at nominal strain $\varepsilon$ of 0.1 (upper) and 0.2 (lower) for the as-quenched model (left), the pressure-promoted thermal rejuvenation model (centre) and the pure thermal rejuvenation model (right). (b) Nominal stress-strain relations along the loading direction ( $z$ direction) during uniaxial loading tests. Black, red and green curves represent the nominal stress-nominal strain relation of the as-quenched model, the pressure-promoted thermal rejuvenation model and the pure thermal rejuvenation model, respectively.

Changes in deformation behaviour by pressure-promoted thermal rejuvenation

To demonstrate the pressure-promoted rejuvenation effect on the plastic performance, we performed uniaxial tensile simulations for three different $\mathrm{Zr}_{50} \mathrm{Cu}_{40} \mathrm{Al}_{10}$ models: the as-quenched model and the thermally rejuvenated models with and without pressure application, i.e., pressure-promoted thermal rejuvenation model and pure thermal rejuvenation model. Figure 5 a shows the von Mises atomic strain evolution during uniaxial tensile tests with a constant strain rate $\dot{\varepsilon}$ of $10^{8} 1 / \mathrm{s}$ at $300 \mathrm{~K}$. We can see marked strain localisation in the 'as-quenched model', whereas more homogeneous deformations with less strain localisation are observed in the two rejuvenated models (Supplementary Movies S5a, S5b and $\mathrm{S} 5 \mathrm{c}$ ). Thus, the rejuvenation induced by the thermal-pressure loading process can realise more homogeneous deformation than the as-quenched model. Figure $5 b$ represents nominal stress and nominal strain relations along the loading direction ( $z$ direction) during the uniaxial loading tests. The maximum stress and elastic stiffness, slope of the stress-strain relation of the thermally rejuvenated model with pressure application are higher than those of the thermally rejuvenated model without pressure application. Moreover, the flow stress of the pressure-promoted thermal rejuvenation model is higher than those of the other two models. Thus, the pressure-promoted thermal rejuvenation process can realise higher strength and stiffness glass without significant loss of plastic deformability than a pure thermal rejuvenation process. We also conducted simple shear and nanoindentation simulations using the same $\mathrm{Zr}_{50} \mathrm{Cu}_{40} \mathrm{Al}_{10}$ models, which predicts that the excellent elastic and plastic performances of the thermally rejuvenated glass with pressure application is maintained even under different loading and boundary conditions (for the simple shear and nanoindentation simulations see Supplementary Figures S8 and S9, and also Supplementary Movies S8a, S8b, S8c, S9a and S9b).

The MGs constructed under high-pressure condition have rich SRO and MRO, but exhibit homogeneous deformation as shown in Figures 3 and 5 . This trend is interesting, but not intuitive. As we revealed in our previous work, ${ }^{2}$ the pure thermal rejuvenation model shows more homogeneous deformation than as-quenched model. This can be understood by thinking of activation energy of local plastic deformation process, which is often called as shear transformation. ${ }^{28}$ A lower activation energy of the local plastic deformation process leads to more homogeneous deformation under a certain strain rate and temperature because the process is more equally activated everywhere in MG due to the less local stress state change by the activation of each process and then the MG has less opportunity to have a localised deformation, which will grow to shear band. The pure thermal rejuvenation model may have a lower activation energy of the plastic deformation process because the model is already in the high-energy rejuvenated state. The same way of thinking may be applicable also to the pressure-promoted thermal rejuvenation model. The pressure-promoted thermal rejuvenation model is also in a highenergy rejuvenated state, although the structural details are different from the pure thermal rejuvenation model, and thus it may have lower activation energy of the plastic deformation process. Therefore, the pressure-promoted thermal rejuvenation exhibits the homogeneous deformation. To confirm this scenario, we also performed nanoindentation simulations for several different pressure-promoted thermal rejuvenation (or ageing) models; $\mathrm{Cu}_{50} \mathrm{Zr}_{50}(\mathrm{LJ})$ (ageing), $\mathrm{Ni}_{80} \mathrm{P}_{20}$ (rejuvenation) and $\mathrm{Al}_{90} \mathrm{La}_{10}$ (rejuvenation) in addition to $\mathrm{Cu}_{50} \mathrm{Zr}_{40} \mathrm{Al}_{10}$ (rejuvenation). All of the rejuvenated models actually exhibit more homogeneous deformation than that of as-quenched model, whereas the aged $L$ model exhibits less homogeneous deformation (Supplementary Figures S9b, S10b, S11b, S12b; Supplementary Movies S9a, S9b, S10a, S10b, S11a, S11b, S12a and S12b). We should note that, regarding elastic stiffness and maximum load, these are maintained in $\mathrm{Zr}_{50} \mathrm{Cu}_{40} \mathrm{Al}_{10}$ (rejuvenation) or even increased in $\mathrm{Cu}_{50} \mathrm{Zr}_{50}(\mathrm{LJ})$ (ageing), whereas decreased in $\mathrm{Ni}_{80} \mathrm{P}_{20}$ (rejuvenation) and $\mathrm{Al}_{90} \mathrm{La}_{10}$ (rejuvenation). Thus, among these glasses, only $\mathrm{Zr}_{50} \mathrm{Cu}_{40} \mathrm{Al}_{10}$ (rejuvenation) departs from the intuitive trend, that is, rejuvenation usually leads to lower stiffness and strength and ageing leads to higher. The excellent elastic performance of $\mathrm{Zr}_{50} \mathrm{Cu}_{40} \mathrm{Al}_{10}$ (rejuvenation) may arise from the significant increase of the icosahedral SRO by the pressure-promoted thermal rejuvenation (Figure 3a), because the icosahedral SRO structure has higher stiffness. ${ }^{24,27}$ The other rejuvenated $\mathrm{Ni}_{80} \mathrm{P}_{20}$ and $\mathrm{Al}_{90} \mathrm{La}_{10}$ glasses do not exhibit such significant SRO change. See again Supplementary Figure S6. 

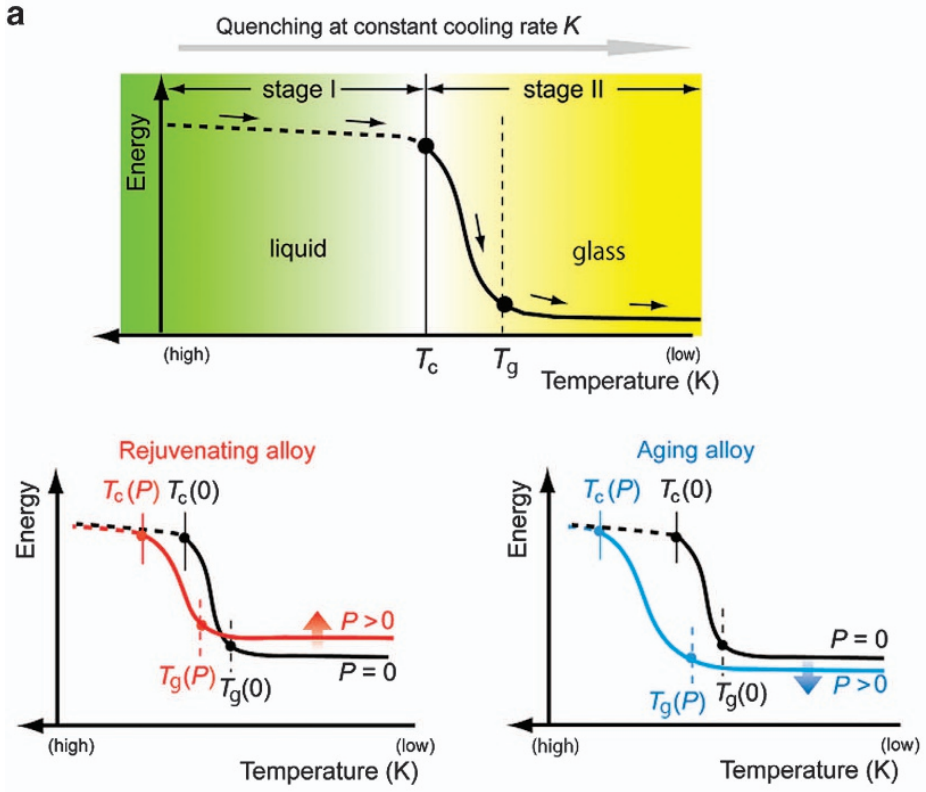

e

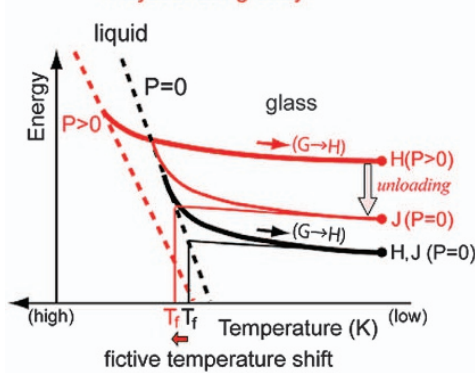

b
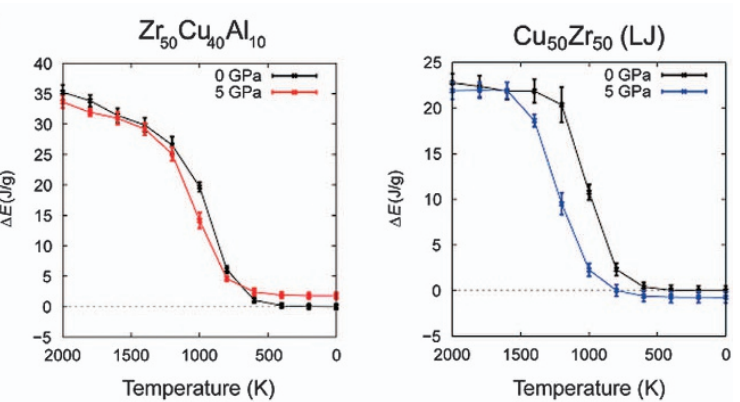

C
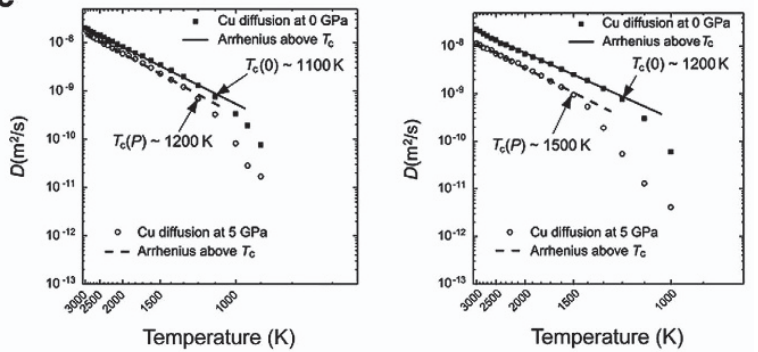

d
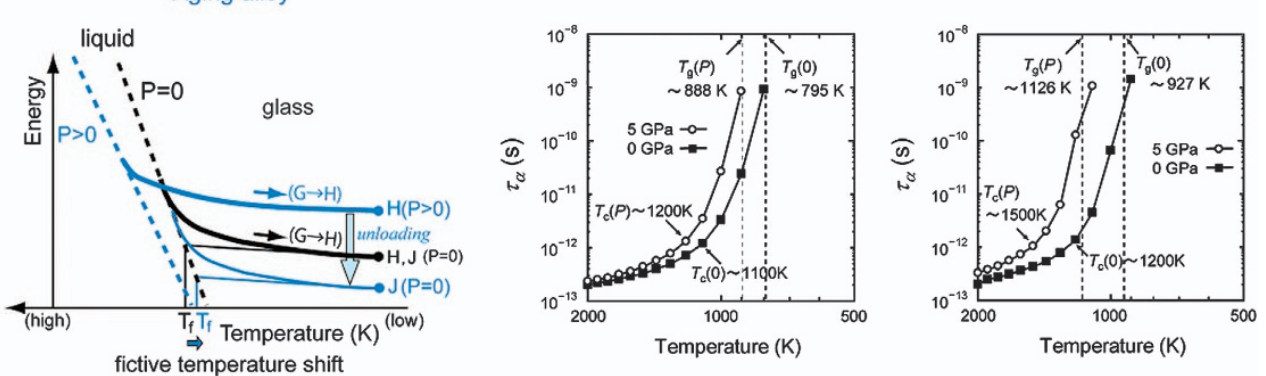

Figure 6. (a) Schematic of inherent structure energy change during quenching process. The horizontal axis represents temperature, which increases from right to left. Upper figure defines cooling stages I and II divided at the critical temperature $T_{c}$ at which transition from Arrhenius behaviour (stage $\mathrm{I} ; T \geqslant T_{\mathrm{c}}$ ) to non-Arrhenius (stage II; $T<T_{\mathrm{c}}$ ) occurs. The lower figures show a typical inherent structure energy change in the quenching process for alloy exhibits rejuvenation (left) or ageing (right). (b) Inherent structure energy change during the quenching process both with and without pressure for the $\mathrm{Zr}_{50} \mathrm{Cu}_{40} \mathrm{Al}_{10}$ alloy (left) and for $\mathrm{Cu}_{50} \mathrm{Zr}_{50}$ (LJ) alloy (right). A crossover between the two curves can be seen in $\mathrm{Zr}_{50} \mathrm{Cu}_{40} \mathrm{Al}_{10}$, whereas no crossover in $\mathrm{Cu}_{50} \mathrm{Zr}_{50}$ (LJ). Details are mentioned in Supplementary Figure S14. (c) The left and right figures show the pressure effect on the temperature-dependent Cu diffusivity of $Z_{50} \mathrm{Cu}_{40} \mathrm{Al}_{10}$ and $\mathrm{Cu}_{50} \mathrm{Zr}_{50}$ ( $\mathrm{LJ}$ ), respectively. (d) The left and right figures show the pressure effect on the temperature-dependent $\alpha$-relaxation time of $\mathrm{Zr}_{50} \mathrm{Cu}_{40} \mathrm{Al}_{10}$ and $\mathrm{Cu}_{50} \mathrm{Zr}_{50}(\mathrm{LJ})$, respectively. In the figures, the critical temperature $T_{\mathrm{c}}$ detected by the diffusively analysis (c) is indicated. The glass transition temperature $T_{\mathrm{g}}$ is also denoted by the vertical dotted lines; $T_{\mathrm{g}}=795 \mathrm{~K}(0 \mathrm{GPa}), 888 \mathrm{~K}(5 \mathrm{GPa})$ for $\mathrm{Zr}_{50} \mathrm{Cu}_{40} \mathrm{Al}_{10}, T_{\mathrm{g}}=927 \mathrm{~K}(0 \mathrm{GPa}), 1,126 \mathrm{~K}(5 \mathrm{GPa})$ for Cu $\mathrm{Cu}_{50} \mathrm{Zr} \mathrm{r}_{50}(\mathrm{LJ})$ ). (e) Schematic of change in the fictive temperature $T_{\mathrm{f}}$ due to pressure in the quenching process for alloys exhibits rejuvenation (left) and ageing (right). The vertical and horizontal axes are energy and temperature, respectively. Thick black curves represent the energy profile in $\mathrm{G} \rightarrow \mathrm{H}$ at $P=0 \mathrm{GPa}$, whereas thick red (left) and blue (right) curves represent that at $P>0 \mathrm{GPa}$. Broken lines represent the energy profile of the equilibrium liquid, whereas solid curves always represent non-equilibrium liquid or glass state. Energy of state $\mathrm{H}$ is reduced to state $\mathrm{J}$ by unloading process $(\mathrm{H} \rightarrow J)$. The thin red (or blue) curves are fictitious cooling curves at $P=0 \mathrm{GPa}$ that reach the glass state $\mathrm{J}$ after unloading in actual pressure-promoted thermal rejuvenation (or ageing) process. We defined the fictive temperature as the intersection point between the equilibrium liquid line of $P=0 \mathrm{GPa}$ and the tangent line of the fictitious cooling curve, which ends state $J$ obtained under $P=0$ or $P>0$ conditions and has the same tangent with the cooling glass curve of $P=0$ condition at state $J$. The fictive temperature $T_{\mathrm{f}}$ of pressurepromoted thermal rejuvenation (left) and ageing (right) are represented by red and blue characters, respectively.

\section{DISCUSSION}

To understand the interesting dependency of the effects of pressure on the alloy composition seen in Figure 2, we discuss structural relaxation in the quenching process $(G \rightarrow H)$ both with and without the effects of pressure below. Figure $6 a$ is a schematic of the change in the inherent structure energy ${ }^{29}$ of an alloy system during the cooling process from far above $T_{\mathrm{g}}$ to $0 \mathrm{~K}$, under a constant cooling rate $K$. The inherent structure energy is the energy of inherent structure defined above. Figure $6 \mathrm{~b}$ shows the actual change in the inherent structure energy of the $\mathrm{Zr}_{50} \mathrm{Cu}_{40} \mathrm{Al}_{10}$ and $\mathrm{Cu}_{50} \mathrm{Zr}_{50}(\mathrm{LJ})$ systems. In Figure $6 \mathrm{~b}$, we may reasonably define a transition temperature $T_{\mathrm{c}}$ as a kink temperature on the curve of the inherent energy change. Above the transition temperature $T_{\mathrm{c}}$ (quenching stage $\mathrm{l} ; T \geqslant T_{\mathrm{c}}$ ), the system can be always in an equilibrium liquid state because of very short relaxation time of the high-temperature liquid. Below $T_{\mathrm{c}}$ (quenching stage II; $T<T_{\mathrm{c}}$ ), owing to a lesser free volume, a finite activation energy for atomic structure relaxation is expected, leading to a finite relaxation time competes against the time of cooling process specified by the cooling rate. To examine the physical meaning of $T_{c}$, we computed the temperature-dependent diffusivity $D$ and a-relaxation time $\tau_{\mathrm{a}}$ of the $\mathrm{Zr}_{50} \mathrm{Cu}_{40} \mathrm{Al}_{10}$ and $\mathrm{Cu}_{50} \mathrm{Zr}_{50}$ (LJ) glasses, as shown in Figure $6 \mathrm{c}$,d. Above $T \sim 1,100 \quad(P=0 \mathrm{GPa})$ and $T \sim 1,200 \mathrm{~K}(P=5 \mathrm{GPa})$ in the case of $\mathrm{Zr}_{50} \mathrm{Cu}_{40} \mathrm{Al}_{10}$ and $T \sim 1,200 \mathrm{~K}(P=0 \mathrm{GPa})$ and $T \sim 1,500 \mathrm{~K}$ $(P=5 \mathrm{GPa})$ in the case of $\mathrm{Cu}_{50} \mathrm{Zr}_{50}(\mathrm{LJ})$, the diffusion kinetics have a 
constant activation enthalpy for diffusion $\Delta G_{0}$, and obeys Arrhenius-type behaviour $D(T) \propto \exp \left(\frac{\Delta G_{0}}{k_{\mathrm{B}} T}\right)$, where $k_{\mathrm{B}}$ is the Boltzmann constant. In contrast, below these temperatures, the diffusion kinetics deviate from Arrhenius-type linear behaviour, indicating a change in the activation enthalpy for diffusion. Because these critical temperatures agree well with $T_{c}$ 's obtained from the kink in Figure $6 \mathrm{~b}, T_{\mathrm{c}}$ could be understood as a transition temperature between Arrhenius and non-Arrhenius behaviours in diffusivity. ${ }^{30}$ Because a higher compressive pressure mechanically reduces the free volume, the pressure actually slows down the diffusion in both the alloys over the whole temperature range, as shown in Figure $6 \mathrm{c}$, and these $T_{\mathrm{c}}$ tends to be higher, i.e., $T_{\mathrm{c}}(P)>T_{\mathrm{c}}(0)$ for $P>0$, as shown in Figure $6 \mathrm{~b}-\mathrm{d}$. It is worth noting that the same trend can be found in the pressure dependency of $T_{\mathrm{g}}{ }^{31}$ (Supplementary Figure S2). In Figure 6d, a-relaxation time $\tau_{a}$ also drastically increases with decreasing temperature at the temperatures ranging from $T_{\mathrm{g}}$ to $T_{\mathrm{c}}$. This is consistent with the diffusivity behaviour in Figure $6 c$. It should be noted that if the annealing time is too short compared with a typical relaxation time, such as a-relaxation time $\tau_{\mathrm{a}}$, the rejuvenation/ageing during the thermal rejuvenation are not sufficiently proceed regardless of with and without pressure application. ${ }^{2}$ The $1 \mathrm{~ns}$ annealing time used in this study is equivalent to or even longer than a-relaxation time $\tau_{\alpha}$ at temperatures $T>T_{\mathrm{g}}$ (necessary temperature condition for the rejuvenation; see Figure $1 \mathrm{~b}$ ) as shown in Figure $6 \mathrm{~d}$. Thus, the $1 \mathrm{~ns}$ annealing time is long enough and does not affect on the rejuvenation.

The final inherent structure energy per atom of the state after the quenching process is completed (i.e., state $\mathrm{H}$ ) at a given $P$ can be expressed as

$$
E_{\mathrm{H}}(P)=E_{\text {stage } \mathrm{I}}\left(P, T_{\mathrm{c}}(P)\right)+K^{-1} \int_{T_{\mathrm{c}}(P)}^{0} \dot{E}(P, T) \mathrm{d} T,
$$

where $K=\mathrm{d} T / \mathrm{d} t$ with $t$ being time. Here $\dot{E}$ is the rate of change of the inherent structure energy and $E_{\text {stage }}\left(P, T_{\mathrm{c}}(P)\right)$ is the inherent structure energy at the end of stage I (i.e., at the beginning of stage II). Hereafter, the inherent structure energy will be simply referred to as the 'energy'. The degree of rejuvenation is estimated from the final energy difference $\Delta E(P)=E_{\mathrm{H}}(P)-E_{\mathrm{H}}(0)$, which can also be expressed as the sum of $(I)$ the energy difference at the end of stage I (i.e., at the beginning of state II) $\Delta E_{\text {stage I }}(P)$ between the $P>0$ and $P=0 \mathrm{GPa}$ conditions and (II) the difference in the total energy change in stage II $\Delta E_{\text {stage } \|}(P)$ between the $P>0$ and $P=0 \mathrm{GPa}$ states, with

$$
\Delta E(P)=\Delta E_{\text {stage I }}(P)+\Delta E_{\text {stage II }}(P),
$$

where

$$
\begin{aligned}
& \Delta E_{\text {stage I }}(P)=E_{\text {stage I }}\left(P, T_{\mathrm{c}}(P)\right)-E_{\text {stage I }}\left(0, T_{\mathrm{c}}(0)\right) \\
& \Delta E_{\text {stage II }}(P)=K^{-1} \int_{T_{\mathrm{c}}(P, T)}^{0} \dot{E}(P, T) d T-K^{-1} \int_{T_{\mathrm{c}}(0, T)}^{0} \dot{E}(0, T) \mathrm{d} T .
\end{aligned}
$$

From the equation and also schematic illustration shown in Figure $6 a$, we can find the following five factors can dominate the final energy difference $\Delta E(P)$. First, there are three factors in the stage I $\left(T \geqslant T_{\mathrm{c}}\right)$. (i) Temperature dependency of the energy. Usually there exists temperature dependency of liquid energy, and highertemperature liquid tends to have higher energy, ${ }^{32}$ i.e., $\partial E(P) / \partial T>0$ $\left(T \geqslant T_{c}(P)\right)$, although alloy dependency can be found as shown in Figure $6 \mathrm{~b}$. Larger temperature dependency leads to larger $\Delta E_{\text {stage }}$, and then contributes to enhance the rejuvenation. (ii) Pressure dependency of energy at $T \geqslant T_{\mathrm{c}}(P)$. The change of energy by pressure can also contributes to the degree of rejuvenation $\Delta E(P)$ via changing $\Delta E_{\text {stage }}(P)$, but usually the pressure dependency should be small, actually error bars of the data with and without pressure are overlapping as seen in Figure $6 \mathrm{~b}$ and Supplementary Figure $\mathrm{S} 13 \mathrm{a}$, because the liquid states at $T \geqslant T_{\mathrm{c}}(P)$ should be always well equilibrated regardless of with and without pressure application. (iii) Pressure dependency of $T_{\mathrm{c}}(P)$. The $T_{\mathrm{c}}$ has not only a strong pressure dependency but also a strong alloy dependency as seen in Figure $6 c, d$ and Supplementary Figure S13b, because it should be closely related to the potential energy surface that determines the starting temperature of the a-relaxation. ${ }^{32}$ The higher $T_{c}(P)$ causes a-relaxation to be initiated at higher temperature, then a rapid energy reduction occurs at earlier stage of the cooling process but below $T_{c}$. As a result the higher $T_{c}(P)$ enhances more ageing. Second, there are two factors in the stage II $\left(T<T_{\mathrm{c}}\right)$. (iv) Pressure dependency of the relaxation time constant (energy reduction rate), which is the slope of the energy curve in Figure 6b, i.e., $\partial E(P) / \partial T\left(T<T_{c}(P)\right)$. Usually the relaxation time becomes longer and thus the energy reduction rate becomes slower with increasing pressure as seen in Figure $6 b-d$ and Supplementary Figure S13a,b, because compressive pressure induces densification (see volume change in Supplementary Figure S13c, induces a less space for moving atom, may effectively suppress the local atomic shuffling and diffusion. (v) Pressure dependency of $T_{\mathrm{g}}(P) . T_{\mathrm{g}}(P)$ is related to the temperature at which the energy reduction rate significantly slows down as shown in Figure $6 \mathrm{~b}, \mathrm{~d}$ and Supplementary Figure $13 \mathrm{Sa}, \mathrm{b}$. The pressure dependency of $T_{g}(P)$ also has a strong alloy dependency as shown in Figure $6 \mathrm{~d}$. The reason should be the same as that of pressure dependency of $T_{c}(P)$. Hence, the factors (i), (iv) and (v) enhance the rejuvenation, whereas the factor (iii) enhances the ageing. Because of the factor (iii), the energy curve under compressive pressure application is always below that of zero pressure at temperatures just below $T_{c}(P)$. Therefore, the two energy curves (i.e., with and without pressure) should have a crossover to realise the rejuvenation eventually. As shown in Figure $6 b$, we actually observed the crossover between the energy-temperature curves both with and without pressure for the $\mathrm{Zr}_{50} \mathrm{Cu}_{40} \mathrm{Al}_{10}$, but not for the $\mathrm{Cu}_{50} \mathrm{Zr}_{50}$ (LJ) (details are given in Supplementary Figure S14). As the crossover temperature increases with increasing the $\partial E(P) / \partial T>0\left(T \geqslant T_{c}(P)\right)$ (factor (i)), the energy reduction rate (factor (iv)) and/or $T_{\mathrm{g}}(P)$ (factor $(\mathrm{v})$ ), and decreases with increasing $T_{\mathrm{c}}(P)$ (factor (iii)), the pressure induces two competing effects in the rejuvenation; (A) the factors (i), (iv) and (v), and (B) the factor (iii). In the case of $\mathrm{Cu}_{50} \mathrm{Zr}_{50}(\mathrm{LJ})$, the latter effect (B) overcomes the former effect $(A)$, results in the pressure induces ageing. In contrast, in the case of $\mathrm{Zr}_{50} \mathrm{Cu}_{40} \mathrm{Al}_{10}$, as $T_{\mathrm{c}}$ increase is much smaller than that of $\mathrm{Cu}_{50} \mathrm{Zr}_{50}$ (LJ) (Figure $6 \mathrm{c}$ ), the former effect overcomes the later effect, results in the pressure induces rejuvenation. Therefore, rejuvenation and ageing by thermal-pressure loading process can be predicted from these factors. It should be noted that all of the data determine that these factors are obtainable and predictable by $\mathrm{MD}$ simulation based on certain potential description of target alloy system as we performed above.

To clarify underlying physics of the pressure-promoted thermal rejuvenation process and characteristics of the pressure-promoted thermally rejuvenated glass, we additionally conducted energy decomposition analyses, which may provide origin of the pressure-promoted higher energy state (rejuvenation) of $\mathrm{Zr}_{50} \mathrm{Cu}_{40} \mathrm{Al}_{10}$ and lower-energy state (ageing) of $\mathrm{Cu}_{50} \mathrm{Zr}_{50}$ (LJ) from energetics and geometrical ordering viewpoints (Supplementary Figures S15 and S16). We found that in the pressure-promoted thermally rejuvenated and aged glass models, the icosahedral SRO has a significant lower-energy state than the other local structures as similar to the conventional MGs constructed under zero pressure (Supplementary Figure S16). Thus, in the energetics and geometrical ordering viewpoints, the high-energy state of the rejuvenated $\mathrm{Zr}_{50} \mathrm{Cu}_{40} \mathrm{Al}_{10}$ glass is attributed to the pressurepromoted significant energy increment of the local structures with lower ordering (i.e., non-icosahedral local structures) as schematically shown in Figure 4. The energy increment of the local 
structure with lower ordering is a result of the less diffusive and mechanical relaxation during the cooling process under the compressive pressure as discussed above Supplementary Figure S17). On the other hand, in the pressure-promoted thermally aged $\mathrm{Cu}_{50} \mathrm{Zr}_{50}$ (LJ) glass, the energy increment of the non-icosahedral local structures is not so significant (Supplementary Figure S15), because a mechanical relaxation (atomic rearrangement) unfortunately may proceed in the $\mathrm{LJ}$ central force field under the compressive pressure, whereas the diffusive relaxation is well suppressed. As a results, the total energy decreases in the $\mathrm{Cu}_{50} \mathrm{Zr}_{50}$ (LJ) glass.

It is worth noting that the less relaxation induced by pressure application may have great benefit also to the glass-forming ability. It effectively prevents a crystallisation of alloy during the quenching process, and thus may improve the apparent glassforming ability of alloy. We should also note that although such a high cooling rate $(K=1.0 \mathrm{~K} / \mathrm{ps})$ was used in the MD simulation because of the time-scale limitations of MD, the above discussion for rejuvenation and ageing should be maintained even at practical cooling rates, because by experiments we have confirmed that the ratio of cooling rate of the final quenching process $(G \rightarrow I)$ to the initial melt-quenching process $(B \rightarrow C)$ is the dominant factor for the degree of rejuvenation and ageing ${ }^{2}$ rather than the absolute cooling rates. In addition, the relationship between energy state and deformation behaviour observed in Figure 5 is also consistent with the critical fictive temperature concept, which identifies the brittle-ductile transition state of MG. ${ }^{33}$ At the fictive temperature $T_{\mathrm{f}}$, the extrapolated glass line intersects the equilibrium liquid line (Figure 6e), thus the $T_{f}$ represents the glass state and then more rejuvenated glass has higher $T_{\mathrm{f}}$. The critical fictive temperature $T_{\mathrm{fc}}$ represents a transition glass state at between glass states showing brittle and ductile behaviours, i.e., a MG having $T_{\mathrm{fc}}>T_{\mathrm{f}}$ is brittle, whereas having $T_{\mathrm{fc}}<T_{\mathrm{f}}$ is ductile. Therefore, the critical fictive temperature concept naturally predicts that the pressure-promoted thermal rejuvenation, which leads to higher $T_{\mathrm{f}}$, can improve the ductility of MGs. As we have shown in Figure 5, the rejuvenated glass having higher $T_{\mathrm{f}}$ exhibits more homogeneous deformation, supporting the validity of the fictive temperature-based discussion of ductility.

We have demonstrated in this study that the feasibility of pressure-promoted thermal rejuvenation for various alloy systems. It was found that rejuvenation is actually enhanced by applying external pressure in many alloy systems, whereas some alloy systems do not exhibit this response. Further, we clarified the underlying physics of pressure-promoted rejuvenation based on diffusivity, a-relaxation, $T_{\mathrm{g}}$ and internal structure analyses. The pressure-promoted rejuvenation is achieved only when the comprehensive influence of three pressure effects on glassforming process, i.e., the effects of temperature-dependent liquid energy, pressure-dependent diffusively slowdown (relaxation slowdown) and pressure-dependent $T_{\mathrm{g}}$ increase, overcomes the effect of pressure-dependent increase in the Arrhenius-nonArrhenius diffusivity transition temperature. Pressure-promoted thermal rejuvenation increases the icosahedral SRO and MRO, even though it leads to an energetically unstable glassy state. This result could change the conventional understanding of the relationship between energy and local structure in MGs, and it strongly indicates the necessity of new energetics of SRO and $\mathrm{MRO}$ under significant pressure. We found that the rejuvenated $\mathrm{Zr}_{50} \mathrm{Cu}_{40} \mathrm{Al}_{10}$ MG made by the pressure-promoted thermal rejuvenation process exhibits greater plastic performance than as-quenched glass, and greater strength and stiffness than glass made by the pure thermal rejuvenation process, and then we believe that this glass rejuvenation engineering technique could introduce a new avenue of research towards the development of glass-state control, and elastic and plastic performance control techniques by thermal processing. Moreover, the pressure effects allow us to increase the upper limit of reachable rejuvenation, which has been restricted by the practical upper limit of a cooling rate in previous rejuvenation-level control techniques with no pressure application. ${ }^{2}$ It also may allow us to extrinsically improve the apparent glass-forming ability of alloys by delaying crystallisation.

\section{MATERIALS AND METHODS}

Models for thermal-pressure loading process shown in Figures 1,2,3 and 6 consist of 10,000 atoms. It is worth noting that a bigger model composed of 100,000 atoms showed quantitatively the same results as the 10,000-atom model (Supplementary Figure S18). Ten different as-quenched models were prepared and then 10 independent thermalpressure loading simulations were conducted to reduce the statistical errors of the data, which are due to the finite model size. Temperature and pressure during quench, anneal and heat process are controlled by the isothermal-isobaric ensemble. ${ }^{34-36}$

Models for uniaxial tensile simulations shown in Figure 5 consist of 1,280,000 atoms by organising 10,000-atom unit models ('As-quench', 'Pressure-promoted thermal rejuvenation' and 'Pure thermal rejuvenation') in an $8 \times 1 \times 16$ array $(x, y, z$ directions, respectively). The as-quenched unit model was constructed via melt-quenching from 3,000 to $0 \mathrm{~K}$ at a relatively slow cooling rate of $0.01 \mathrm{~K} / \mathrm{ps}$. The pressure-promoted thermal rejuvenation and pure thermal rejuvenation unit models were constructed via the thermal-pressure loading process of $P_{\mathrm{a}}=21 \mathrm{GPa}$ and $P_{\mathrm{a}}=0 \mathrm{GPa}$, respectively, with $T_{\mathrm{a}}=1.3 T_{\mathrm{g}}\left(P_{\mathrm{a}}\right)$. In case of $P_{\mathrm{a}}=0 \mathrm{GPa}$, thermal rejuvenation was realised by the relatively fast cooling rate of the final quenching process $(\mathrm{G} \rightarrow \mathrm{H}) 0.20 \mathrm{~K} / \mathrm{ps}$, whereas in case of $P_{\mathrm{a}}=21 \mathrm{GPa}$, the cooling rate of the final quenching process $(\mathrm{G} \rightarrow \mathrm{H})$ is set to $0.01 \mathrm{~K} / \mathrm{ps}$, which is the same as that of the as-quenched unit model, and thus in this cooling rate condition the pressure application is necessary for rejuvenation. The assembled 1,280,000-atom simulation models were heated from 0 to $300 \mathrm{~K}$ at a rate of $1.0 \mathrm{~K} / \mathrm{ps}$ and then relaxed at $300 \mathrm{~K}$ for $1.0 \mathrm{~ns}$ under full (i.e., $x, y$ and $z$ ) periodic boundary and additionally relaxed at $300 \mathrm{~K}$ for $100 \mathrm{ps}$ with partial periodic boundary condition only in $y$ and $z$ (free surface boundary in $x$ ). Using this model, we conducted uniaxial tensile simulations. Note that after the relaxation processes both the 1,280,000-atom simulation models, i.e., pressure-promoted thermal rejuvenation model and pure thermal rejuvenation model, had higher-energy states than the as-quenched model by 9.6 and $10.6 \mathrm{~J} / \mathrm{g}$, respectively. Energy reductions in the relaxation process for totally $1.1 \mathrm{~ns}$ were $<1.0 \mathrm{~J} / \mathrm{g}$, implying that rejuvenated states are maintained during the deformation tests for $2 \mathrm{~ns}$ after the relaxation process.

Diffusivity $D$ is calculated from the mean-square displacement of the atoms. a-Relaxation time $\tau_{a}$ is defined by the time needed for the self-intermediate scattering function at first peak of structural factor decays to $1 / e^{30}$

\section{ACKNOWLEDGEMENTS}

This work is supported by the following funding awards: Grants-in-Aid for Scientific Research in Innovative Area (no. 22102003), Scientific Research (A) (no. 23246025), Challenging Exploratory Research (no. 25630013) and the Elements Strategy Initiative for Structural Materials (ESISM).

\section{CONTRIBUTIONS}

N.M. conducted almost all calculation, Y.-J.W. performed the diffusivity computation, and M.W. performed a-relaxation time analyses and MRO analyses. M.W. and S.O. assisted with data interpretation and designed the paper. S.O. designed and organised the study. All authors discussed the results and participated in writing the paper.

\section{COMPETING INTERESTS}

The authors declare no conflict of interest.

\section{REFERENCES}

1. Struik, L. C. E. Physical Aging in Amorphous Polymers and Other Materials. (Elsevier, Houston, TX, USA, 1978)

2. Wakeda, M., Saida, J., Li, J. \& Ogata, S. Controlled rejuvenation of amorphous metals with thermal processing. Sci. Rep. 5, 10545 (2015). 
3. Meng, F., Tsuchiya, K., li, S. \& Yokoyama, Y. Reversible transition of deformation mode by structural rejuvenation and relaxation in bulk metallic glass. Appl. Phys. Lett. 101, 121914 (2012).

4. Ketov, S. V. et al. Rejuvenation of metallic glasses by non-affine thermal strain. Nature 524, 200-203 (2015)

5. Concustell, A., Méar, F. O., Surinach, S., Baró, M. D. \& Greer, A. L. Structural relaxation and rejuvenation in a metallic glass induced by shot-peening. Philos. Mag. Lett. 89, 831-840 (2009).

6. Saida, J., Yamada, R. \& Wakeda, M. Recovery of less relaxed state in Zr-Al-Ni-Cu bulk metallic glass annealed above glass transition temperature. Appl. Phys. Lett. 103, 221910 (2013)

7. Adachi, N., Todaka, Y., Yokoyama, Y. \& Umemoto, M. Improving the mechanical properties of Zr-based bulk metallic glass by controlling the activation energy for $\beta$-relaxation through plastic deformation. Appl. Phys. Lett. 105, 131910 (2014).

8. Kumar, G., Prades-Rodel, S., Blatter, A. \& Schroers, J. Unusual brittle behavior of Pd-based bulk metallic glass. Scr. Mater. 65, 585-587 (2011).

9. Xi, X. K. et al. Fracture of brittle metallic glasses: brittleness or plasticity. Phys. Rev. Lett. 94, 125510 (2005).

10. Kumar, G., Rector, D., Conner, R. D. \& Schroers, J. Embrittlement of Zr-based bulk metallic glasses. Acta Mater. 57, 3572-3583 (2009).

11. Kumar, G., Hong, X. T. \& Schroers, J. Nanomoulding with amorphous metals Nature 457, 868-872 (2009)

12. Wang, W. H. et al. Effect of pressure on nucleation and growth in the $\mathrm{Zr}_{46.75} \mathrm{Ti}_{8.25} \mathrm{Cu}_{7.5} \mathrm{Ni}_{10} \mathrm{Be}_{27.5}$ bulk glass-forming alloy investigated using in situ x-ray diffraction. Phys. Rev. B 68, 184105 (2003).

13. Cheng, Y. Q., Ma, E. \& Sheng, H. W. Atomic level structure in multicomponent bulk metallic glass. Phys. Rev. Lett. 102, 245501 (2009).

14. Fujita, T. et al. Coupling between chemical and dynamic heterogeneities in a multicomponent bulk metallic glass. Phys. Rev. B 81, 140204 (2010).

15. Mendelev, M. I. et al. Development of suitable interatomic potentials for simulation of liquid and amorphous Cu-Zr alloys. Philos. Mag. 89, 967-987 (2009).

16. Kobayashi, S., Maeda, K. \& Takeuchi, S. Computer simulation of deformation of amorphous $\mathrm{Cu}_{57} \mathrm{Zr}_{43}$. Acta Metall. 28, 1641-1652 (1980).

17. Sheng, H. W., Cheng, Y. Q., Lee, P. L., Shastri, S. D. \& Ma, E. Atomic packing in multicomponent aluminum-based metallic glasses. Acta Mater. 56, 6264-6272 (2008)

18. Sheng, H. W., Ma, E. \& Kramar, M. J. Relating dynamic properties to atomic structure in metallic glasses. JOM 64, 856-881 (2012).

19. Ding, J., Cheng, Y. Q., Sheng, H. W. \& Ma, E. Short-range structural signature of excess specific heat and fragility of metallic-glass-forming supercooled liquids. Phys. Rev. B 85, 060201 (2012).

20. Hirata, A. et al. Geometric frustration of icosahedron in metallic glasses. Science 341, 376-379 (2013)
21. Mendelev, M. I., Srolovitz, D. J., Ackland, G. J. \& Han, S. Effect of Fe segregation on the migration of a non-symmetric $\Sigma 5$ tilt grain boundary in Al. J. Mater. Res. 20, 208-218 (2005).

22. Wang, Z. T., Pan, J., Li, Y. \& Schuh, C. A. Densification and strain hardening of a metallic glass under tension at room temperature. Phys. Rev. Lett. 111, 135504 (2013).

23. Frank, F. C. Supercooling of liquids. Proc. R. Soc. Lond. A Math. Phys. Sci. 215, 43-46 (1952).

24. Wakeda, M. \& Shibutani, Y. Icosahedral clustering with medium-range order and local elastic properties of amorphous metals. Acta Mater. 58, 3963-3969 (2010).

25. Wakeda, M., Shibutani, Y. \& Ogata, S. Atomistic study on medium-range order structures in amorphous metals. J. Soc. Mater. Sci. Japan 64, 156-162 (2015).

26. Ma, E. Tuning order in disorder. Nat. Mater. 14, 547 (2015).

27. Wakeda, M., Shibutani, Y., Ogata, S. \& Park, J. Relationship between local geometrical factors and mechanical properties for $\mathrm{Cu}-\mathrm{Zr}$ amorphous alloys. Intermetallics 15, 139-144 (2007).

28. Argon, A. S. Plastic deformation in metallic glasses. Acta Metall. 27, 47-58 (1979)

29. Stillinger, F. H. \& Weber, T. A. Packing structures and transitions in liquids and solids. Science 225, 983-989 (1984).

30. Jaiswal, A., Egami, T. \& Zhang, Y. Atomic-scale dynamics of a model glass-forming metallic liquid: Dynamical crossover, dynamical decoupling, and dynamical clustering. Phys. Rev. B 91, 134204 (2015).

31. Debenedetti, P. G. Metastable Liquid: Concepts And Principles. (Princeton Univ. Press, Princeton, NJ, USA, 1996).

32. Debenedetti, P. G. \& Stillinger, F. H. Supercooled liquids and the glass transition. Nature 410, 259-267 (2001).

33. Kumar, G., Neibecker, P., Liu, Y. H. \& Schroers, J. Critical fictive temperature for plasticity in metallic glasses. Nat. Commun. 4, 1536 (2013).

34. Nosé, S. A molecular dynamics method for simulations in the canonical ensemble. Mol. Phys. 52, 255-268 (1984).

35. Hoover, W. G. Canonical dynamics: equilibrium phase-space distributions. Phys. Rev. A 31, 1695-1697 (1985).

36. Parrinello, M. \& Rahman, A. Polymorphic transitions in single crystals: a new molecular dynamics method. J. Appl. Phys. 52, 7182-7190 (1981).

37. Shimizu, F., Ogata, S. \& Li, J. Theory of shear banding in metallic glasses and molecular dynamics calculations. Mater. Trans. 48, 2923-2927 (2007).

(C) This work is licensed under a Creative Commons Attribution 4.0 International License. The images or other third party material in this article are included in the article's Creative Commons license, unless indicated otherwise in the credit line; if the material is not included under the Creative Commons license, users will need to obtain permission from the license holder to reproduce the material. To view a copy of this license, visit http://creativecommons.org/licenses/ by/4.0/

(c) The Author(s) 2016

Supplementary Information accompanies the paper on the npj Computational Materials website (http://www.nature.com/npjcompumats) 